Pre- and posttest comparisons were performed on buth water and air estimates (the latter measures the aftereffect of adaptation). Size estimates in the water postlest were significantly smaller than in the pretest $(p<.025$; Wilcoxon, one-tailed). The air posttest estimates were not significantly different from the air pretest judgments. The failure to find significant adaptation as measured by the aftereffect was not unexpected. The aftereffect is known to fade rapidly (Dewar, 1970; Ross et al, in press) and could easily have dissipated while the diver left the pool, removed his diving gear, and ran to the air test site. Although the data clearly indicate distortion of distance under water, a pre- and posttest comparison failed to reveal any adaptation to this distortion. The posttest judgments tended to be larger than pretest judgments, both in water and in air, but these differences were very small and did not approach significance.

The analyses of the pre- and posttest difference scores (in water) at first suggested that, on the average, divers adapted to size but did not alter their distance judgments. These findings are not consistent with the strict form of the size-distance invariance hypothesis, which predicts counteradaptation to distance in proportion to the degree of size adaptation. However, an inspection of the data revealed that the pre- and posttest comparisons may not adequately reflect the results. If some Ss adapted to size (by further distorting distance) and some Ss adapted to distance (by further distorting size), then the average pre- and posttest difference score on each dimension would be appreciably reduced. Therefore, a more appropriate analysis of the relation between size and distance adaptation was performed. A Spearman rank-order correlation on the size and distance adaptation scores (total pre- minus total posttest differences) of the water tests revealed a significant negative correlation (rho $=-.71, \mathrm{p}<.025$ ), indicating that size adirtation was indeed inversely related to distance adaptation. The same analysis for the control Ss showed no relation between these two sets of difference scores $($ rho $=-.17)$. The relation between size and distance adaptation is shown in Fig. 1: divers who adapt on one dimension tend to counteradapt on the other. Of the 11 divers, 8 demonstrated this effect, 6 adapting to size while counteradapting to distance and 2 the other way around. It is noteworthy that the three remaining scores fall in the upper right-hand quadrant of the figure, suggesting that adaptation to both size and distance may be possible.
The correlational analysis suggests that for most divers, adaptation to size and distance does not occur simultaneously. Rather, adaptation on one dimension tends to be accompanied by further distortion of the other. Adaptation to size was accompanied by further distortion of distance and vice versa. Although these findings do not necessarily imply strict proportionality of the size-distance relationship, they do indicate that at least a weak form of size-distance invariance is maintained during adaptation. The results also suggest the hypothesis that adaptation to distortion can have the effect of providing both increased and decreased correspondence between perception and the environment.

\section{REFERENCES}

DEWAR, R. Adaptation to displaced vision: The influence of distribution of practice on retention. Perception \& Psychophysics, 1970. 8, 33-34.
KINNEY. J. A. S.. LLRIA, S. H. \& WEITZMAN, D. O. Responses to the underuater distortion of visual stimuli. LSNSUC Report No.541, L'.S. Naval Submarine Base, Groton, Connecticut, 1968.

LURIA, S. M., \& KINNEY, J. A. S. Underwater vision. Science, 1970, 167, 1454-1461.

LURIA, S. M., KINNEY, J. A. S.. \& WFISSMAN, $S$. Estimates of size and distance undervater. American Journal of Psychology, 1967, 80, 282-286.

ROCK, I. Adaptation to a minified image. Psychonomic Science, 1965, 2, 105-106.

ROCK, I. The nature of perceptual adaptation. New York: Basic Books, 1966.

ROSS, H. E. Water, fog, and the size-distance invariance hypothesis. British Journal of Psychology, 1967, 58, 301-313.

ROSS, H. E. Adaptation of divers to curvature distortion under water. Ergonomics, 1970, 12. (in press).

ROSS, H. E., FRANKLIN, S. S., WELTMAN, G., \& LENNIE, P. Adaptation of divers to size distortion under water. British Journal of Psychology, 1970, 61, 365-373.

STRATTON, G. M. Experimental psychology and culture. New York: Macmillan, 1903.

\title{
TSD and coding in STM
}

\author{
JOHN M. ACKROFF* and RICHARD O. ROUSE, JR. $\dagger$ \\ Williams College, Williamstown, Mass. 01267
}

Ss were asked to listen to a list of words and to identify repeated words upon hearing them. Interspersed with the repetitions were words that were associatively or acoustically related to the repeated words. The intrusion errors were analyzed and the differences across word class found to be highly significant; application of signal-detection theory provided a means of quantifying this difference.

Marshall, Rouse, \& Tarpy (1969) present a summary of previous work in the field of coding strategies in STM. Of special interest for their study, and for the present one, are the works of Conrad $(1962,1964)$ and Wickelgren (1966), which present an acoustical model for STM, and Schwartz \& Rouse (1961), which postulates an associative model.

Broadbent \& Gregory (1963) used

*National Science Foundation Undergraduate Research Participant.

$\uparrow$ Please address requests for reprints to Professor Richard O. Rouse, Jr., Dept of Psychology, Williams College, Williamstown, Mass. 01267. signal-detection theory (TSD) to study attention. Digits were presented to one ear while bursts of noise were presented to the other; application of TSD allowed Broadbent and Gregory to determine the separation between the noise $(\mathrm{N})$ and signal-plus-noise (SN) distributions. Murdock (1965) also used TSD in a memory task, using recognition of paired associates. After being presented with a series of PAs, Ss were to determine whether each of another set of PAs had been seen before; application of TSD allowed Murdock to analyze the types of intrusion errors that were made.

The purpose of this experiment is to 
compare the associative and acoustical models after the fashion of Marshall, Rouse, \& Tarpy (1969) by allowing the Ss to make mistakes that are associatively or acoustically related to the correct response or to make mistakes that are related neither associatively nor acoustically. We shall then apply TSD to the results in order to determine the degree to which each coding strategy is used.

\section{SUBJECTS}

Sixty members of psychology courses at the North Adams State College summer program were used as $\mathrm{Ss}$ for this experiment. ${ }^{1}$ all were told before their classes that they would be taking part in a study of memory, but no specific information about the experiment was given to them.

\section{MATERIALS}

A tape recording was made, consisting of 148 words common in daily usage. ${ }^{2}$ The words were read at the rate of one every $4 \mathrm{sec}$. The first 10 words on the list were buffers (B), chosen at random from the Thorndike \& Lorge (1944) word count. The next 46 words were the 23 pivot words $(\mathrm{P}$, which would be repeated later in the tape) and 23 more Bs, randomly mixed. The remaining 92 words of the list were the second presentation of each of the 23 Ps, the high associate (HA) for each $P$, a nonassociated rhyme $(R)$ for each $P$, and 23 new Bs. All Ps had rates of occurrence of 50 to 100 times per million, and the median frequency of occurrence for the entire list was also in this range (Thorndike \& Lorge, 1944). The mean rate of association for the HAs was 132.8 (Palermo \& Jenkins, 1964). The Bs were all tested to be sure that none of them was associatively or acoustically related to any of the Ps, HAs, Rs, or other Bs.

\section{PROCEDURE}

Ss were instructed to listen to the list and were told that some of the words would be repeated and some of them would not. They were told that for each word they were to indicate on their answer sheets whether or not the word had been previously heard and also to indicate their confidence by writing a 1 (very unsure), 2 , 3,4 , or 5 (very sure) for each word. The instructions were recorded on the tape, so that they would be the same for each class; after the instructions were given, an example was presented to ensure that all Ss understood the task, and then the tape was stopped so that any questions the Ss might have could be answered.

\section{RESULTS}

Only intrusion errors (words that $S$ thought he had previously heard but which were in fact not repeated) were scored. The mean frequency of errors was 4.53 for HAs, 2.05 for Rs, and 1.97 for Bs. An analysis of variance showed the interaction among the groups to be highly significant $[F(4,236)=49.152, \quad p<.001] . \quad$ Further analysis using $t$ tests showed that HA intrusion errors were made significantly more frequently $[\mathrm{t}(118)=7.569$, $p<.001]$ than $R$ intrusion errors, but that $R$ intrusion errors were not made significantly more frequently than $B$ intrusion errors $[t(118)=0.357, p>.05]$.

The data were then analyzed using the procedures of TSD; the value of $d^{\prime}$ (representing the separation of the means of the $N$ and $S N$ distributions and measured in standard deviations) was found to be $1.5 \sigma$ for the $\mathrm{Ps}, 0.3 \sigma$ for the $\mathrm{HAs}$, and $0.01 \sigma$ for the Rs, using the Bs for the $\mathrm{N}$ distribution in all cases.

\section{DISCUSSION}

The results of this experiment support the finding of Marshall et al (1969) that the coding strategy used for words is associative rather than acoustical. Conrad, Freeman, and Hull (1965) point out that the coding strategy which the $\mathrm{S}$ adopts is in part dependent on the nature of the material to be learned; in the Conrad $(1962,1964)$ and Wickelgren $(1965,1966)$ studies, the material was single letters, which are low in meaningfulness and associative value and, therefore, the $\mathrm{Ss}$ adopted coding strategies that were primarily acoustical. In the Marshall et al study (1969); as well as in this study, the material was words, so that Ss could make a choice between an associational and an acoustical, or any other, kind of strategy; in each case the associational method was chosen.

The use of TSD allows this to be shown in another way. The decreasing values of $d^{\prime}$ as the $\mathrm{SN}$ distribution is changed from $\mathrm{P}$ to HA to $R$, while the $N$ distribution is represented by $B$, show that the two distributions come closer together; in the case of the $\mathbf{R}$ and $\mathrm{B}$ distributions, the two curves almost completely overlap, since the separation of the means is only $0.01 \sigma$ and the curves are assumed to be of equal variance. This implies that in the recognition process very little distinction is made between Rs and $\mathrm{N}$, somewhat more between HAs and $\mathrm{N}$, and still more between $P_{S}$ and $N$. Since such a small distinction is made between $R$ and $N$, we can conclude not only that the coding strategy that is adopted is primarily associative but also that an acoustical strategy is almost never used.

To put this in terms of processes, we could say that when a word is presented, a trace is formed. Somewhat weaker traces are formed on an associative dimension, and traces that are weaker still are formed on an acoustical dimension. The traces of the words themselves are strong enough so that the recognition pdf (probability density function) for these words is separated from the $\mathrm{N}$ pdf by $1.5 \sigma$. The traces on the associative dimension form a recognition pdf which is separated from the $\mathrm{N}$ pdf by only $0.3 \sigma$. The traces on the acoustical dimension form a recognition pdf which is only barely separated $\left(d^{\prime}=0.01\right)$ from the $N$ pdf. In order for $N$ of mean strength to be mistakenly recognized as a $P$, then, the $P$ for which it is being mistaken must have a trace strength $1.5 \sigma$ weaker than the mean $P$ trace strength; for a mean-strength $\mathrm{HA}$ to result in an intrusion error, the $\mathrm{HA}$ must be mistaken for a $P$ whose trace strength is $1.2 \sigma(=1.5 \sigma-0.3 \sigma)$ lower than the mean $P$ trace strength, and for a mean-strength $R$ intrusion error to occur, the $\mathrm{R}$ must be substituted for a $\mathrm{P}$ whose trace strength is $1.49 \sigma$ less than the mean $\mathbf{P}$ trace strength.

\section{REFERENCES}

BROADBENT, D. E., \& GREGORY, M. Division of attention and the decision theory of signal detection. Proceedings of the Roy al Society of London, Series B, 1963, 158, 222-231.

CONRAD, R. An association between memory errors and errors due to acoustic masking speech. Nature, 1962, 193, 1314-1315.

CONRAD, $R$. Acoustic confusions in immediate memory. British Journal of Psychology, 1964, 55, 75-84.

CONRAD, R., FREEMAN, P. R., \& HULL, A. J. Acoustic factors versus language factors in short-term memory. Psychonomic Science, $1965,3,57-58$.

MARSHALL, J. F., ROUSE, R. O., \& TARPY, $R$. M. Acoustic versus associative models of short-term memory coding. Psychonomic Science. 1969, 14, 54-55.

MURDOCK, B. B. Signal-detection theory and short-term memory. Journal of Experimental Psychology, 1965, 70, 443-447.

PALERMO, D. S., \& JENKINS, J. J. Word association norms: Grade school through college Minneapolis: University of Minnesota Press, 1964.

SCHWARTZ, F., \& ROUSE, R. O. The activation and recovery of associations. Psychological Issues, 1961, 3, 1-141.

THORNDIKE, E. L., \& LORGE, I. The teacher's word book of 30,000 words. New York: Bureau of Publications, Teachers College, Columbia University, 1944.

WICKELGREN, W. A. Acoustic similarity and intrusion errors in short-term memory. Journal of Experimental Psychology, 1965, 70, 102-108.

WICKELGREN, w. A. Short-term recognition memory for single letters and phonemic similarity in RI. Quarterly Journal of Experimental Psychology, 1966, 18, 55-62. NOTES

1. Thanks are hereby gratefully extended to Dr. Wiltiam Anderson for arranging the schedule and to Mrs. Lila Anderson, Mr. George Cross, and Mr. Samuel Gomez for giving us class time.

2. We are grateful to Joan Hertzberg, also an NSFURP, for making the tape and to Mrs. D. Giusti for help in analyzing the data. 